\title{
Tran-Radial Percutaneous Coronary Intervention (PCI) is Safe and Alternative to Conventional Trans-Femoral Approach: Our Experiences at Apollo Hospitals Dhaka
}

\author{
AQM Reza, AHMW Islam, S Munwar, S Talukder, T Ahmed, A H Bhuiyan, AB Siddique, \\ R Masud, SR Shohel, MS Alam, A Miah \\ Dept. of Invasive cardiology, Apollo Hospitals Dhaka
}

\begin{abstract}
:
Keywords: PCI, Trans radial, Transfemoral.

Background: Aim of the study was to assess the safety of the trans-radial Percutaneous coronary intervention (PCI) than conventional Trans-femoral approach by using either Bare-metal stents (BMS) and or Drug Eluting Stent (DES) like Sirolimus-eluting or Paclitaxel-eluting stent. Also to see its safety in regards of procedural time, quick mobilization, less complication and less radiation exposure.

Methods: Total 117 patients were randomized from a total of 538 patients who had PCI at our center in the quantifying period. Total 130 stent deployed in 117 patients. Among the patients, Male: 100 and Female:17. Mean age were for Male: 55yrs, for Female:57yrs . Associated Coronary artery disease (CAD) risk factors were Dyslipidemia, High Blood pressure, Diabetes Mellitus, Positive FH for CAD and Smoking (all male).

Results: Our study shows 21.7\% had trans-radial PCI. Among the study group; 72 (61.5\%) were Dyslipidemic, 75 (64.1\%) were hypertensive: 47(40.2\%) patients were Diabetic, FH 29 (24.8\%) and $33(33 \%)$ were all male smoker. Female patients were more obese (BMI M 27: F 29) and developed CAD in advance age. Common stented territory were LAD 51 (43.6\%) followed by RCA 41 (35\%) and LCX $27(23 \%)$. Average length and diameter of stented vessel were almost same in all territory. Territory wise multiple or overlapping stenting was done in LAD 3 (6 stents), RCA 1(2stents), LCX 4 (10 stents). Stent used: BMS 37 (28.5\%), Sirolimus 41(31.5\%), Paclitaxel 27 (21\%), Everolimus 22 (16.9\%), Biolimus $2(1.5 \%)$ and Zotarolimus 1(0.75\%). Less Procedural time, reduced radiation exposure, no procedural complication and overall better patient comfort were observed.

Conclusion: Our study has revealed that trans-radial PCI is safe with reduced radiation exposure, quick mobilization of patient and no procedural complication in all the case, indicates it can be done routinely as an alternative to conventional Trans-femoral approach.
\end{abstract}

(Cardiovasc.j. 2012; 5(1): 57-61)

\section{Introduction:}

Since, the catheterization of a human heart was first performed by Werner Frossmann in 1929, access site practice has undergone considerable evolution and technical refinement. ${ }^{1}$ The most common access site use is Trans femoral approach (TFA) for any interventional procedure specially in case of coronaries. Radial artery (RA) access for CAG was first described by Campeau, ${ }^{2}$ with coronary intervention performed shortly after. Improvement in technique and equipments have resolved this problem and more recent success rates are comparable to those of femoral access. ${ }^{3}$
Several Meta-analysis of randomized trials confirmed that radial access is associated with significant reduction in access site bleeding. Prevention of excess bleeding by radial approach, many has explained its association of reduction in significant bleeding related complication and thereby mortality in trans-radial approach. ${ }^{4}$ Data on performed PCI by radial approach in Bangladeshi stent era is yet to be available. Therefore, we have analyzed this non randomized prospective cohort study, in order to see radial access superiority than femoral approach in our patient population.

Address of Correspondence : Dr. A H M Waliul Islam, Dept. of Interventional and Invasive cardiology, Apollo Hospitals Dhaka, Bangladesh. email- ahmwislam@apollodhaka.com 


\section{Methods:}

Total 117 patients were selected in non-randomized method from a total of 538 patients who had PCI at our center in the quantifying period. Total 130 Stents were deployed in 117 patients. Among the patients, Male: 100 and Female: 17. Mean age were for Male: $55 \mathrm{yrs}$, for Female: $57 \mathrm{yrs}$. Associated CAD risk factors were Dyslipidemia, High Blood pressure, Diabetes Mellitus, Positive FH for CAD and Smoking (all male).

Procedure: CAG was performed as per standard protocol. Individual discretion was applied as per operator's choice, depending on patient's problem. PCI either were done with BMS or DES. DES included Cypher (Cordis, USA) and TAXUS (Boston Scientific, USA). The BMS included MultilinkVision, Zeta (Guidant, USA) and micro-Driver (Medtronic, USA) and Genous (Orbus, Netherlands). Coronary angioplasty was performed according to standard rules. Predilatation was optional before stent implantation with a shorter balloon to avoid geographic miss. A successful procedures was defined as TIMI-3 antegrade flow, and $<20 \%$ residual stenosis in two orthogonal views. Post-deployment dilation was performed at high inflation pressure in all patients.

\section{Drug Therapy}

All the patients received Aspirin $300 \mathrm{mg} /$ day and Clopidegrol as a loading dose $300 \mathrm{mg}$ prior to PCI and continued for 3-6 months and received atorovastatin along with standard Medical management for CAD. During the procedure, an intravenous heparin bolus (100IU/Kg) and GP IIb/ IIIa receptor blocker Integrilin were administered as required. The use of GP IIb/IIIa Receptor blocker was recommended as per protocol. Quantitative angiographic measurements of the target lesion were obtained in order to deploy correct size stent. In the event of chest pain, postprocedural ECG and CPK were measured and compared with the baseline. Check angio were taken, whenever indicated.

Data: Data were presented as mean \pm SD with percentage. Significant lesion was defines as stenosis as greater than $70 \%$ narrowing with clinical symptoms. Patient were selected and analyzed from those who underwent routine coronary angiogram for further evaluation of their coronary status. Most of the patient population in this study, had PCI either with bare metal stent or drug eluting stent

\section{Results:}

Our study shows, 117 patients (21.7\%) out of 538 patients had trans-radial PCI for the designated period of 2008-2011.

Table-I

Demographic Profile of patient

\begin{tabular}{lcc}
\hline & Male & Female \\
\hline Number & 100 & 17 \\
Age $(\mathrm{yrs})$ & $54.5 \pm 11.8$ & $56.7 \pm 7.5$ \\
BMI $\left(\mathrm{kg} / \mathrm{m}^{2}\right)$ & $26.9 \pm 4.7$ & $29.3 \pm 2.5$ \\
SBP $(\mathrm{mmHg})$ & $134 \pm 17.7$ & $131 \pm 13.6$ \\
DBP $(\mathrm{mmHg})$ & $75.6 \pm 11.1$ & $78.9 \pm 6.0$ \\
No.of Risk Factor & $2.1 \pm 0.6$ & $2.2 \pm 0.7$ \\
\hline
\end{tabular}

Data were presented as Mean \pm SD

Table-II

Average size of Stent used with inflation pressure

\begin{tabular}{lccc}
\hline & $\begin{array}{c}\text { Length } \\
(\mathrm{mm})\end{array}$ & $\begin{array}{c}\text { Diameter } \\
(\mathrm{mm})\end{array}$ & $\begin{array}{c}\text { Inflation } \\
\text { Pressure(ATM) }\end{array}$ \\
\hline $\mathrm{LAD}$ & $23.3 \pm 8.1$ & $3.2 \pm 0.4$ & $14.8 \pm 2.6$ \\
$\mathrm{LCX}$ & $17.5 \pm 6.0$ & $3.0 \pm 0.5$ & $15.5 \pm 2.6$ \\
RCA & $20.7 \pm 6.3$ & $3.1 \pm 0.4$ & $14.7 \pm 2.1$ \\
\hline
\end{tabular}

Data were presented as Mean \pm SD

Table-III

PCI procedures

\begin{tabular}{lc}
\hline Dye Used(ml) & $49.5 \pm 8.9$ \\
Fluoro Time(Sec) & $193 \pm 23.4$ \\
Procedural time for SVD(Min) & $15.3 \pm 5.6$ \\
Fluro-used (miliGray) & $4727.5 \pm 343$ \\
Hospital stay(Hr & $36.0 \pm 6.0$ \\
Full Mobilization (Hrs) & $6.0 \pm 2.0$ \\
\hline
\end{tabular}

Table-IV

Complication of Trans-radial PCI

\begin{tabular}{lc}
\hline Painful RA Spasm & $2 \%$ \\
Hamatoma & $<1 \%$ \\
Haemorrhage & $0 \%$ \\
Haemostasis & Done by pressure \\
& bandage $<2 \mathrm{hrs}$ \\
Echymosis & $<1 \%$ \\
Patient Choice & $100 \%$ prefer \\
\hline
\end{tabular}


Table 1. shows the profile of studied population. Female patients were more obese (BMI; M 27: F 29) and developed CAD in advance age. Table 2. shows the average size of stent used. LAD territory lesion was longer, and followed by RCA and LCX. Therefore LAD territory needs longer stent, then RCA and LCX. Table 3. shows the procedure time, contrast used and hospital stay. Average dye used 50ml, Fluro times $193 \mathrm{sec}$, procedural time $15 \mathrm{~min}$, Fluro used $4727 \mathrm{mGy}$, Hospital stay $36 \mathrm{hrs}$ and full mobilization 6 hrs. Table 4. procedural complication. Painful RA spasm in $2 \%$, Haematoma $<1 \%$, Echymosis $<1 \%$, Haemostasis done by pressure bandage $<2 \mathrm{hrs}$.

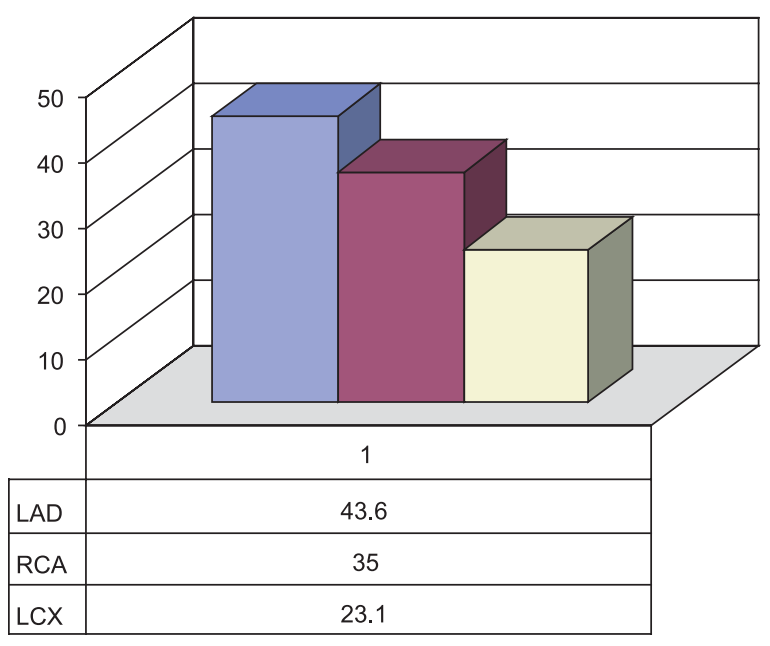

Fig.-1: Percentage distribution of Stented Territory

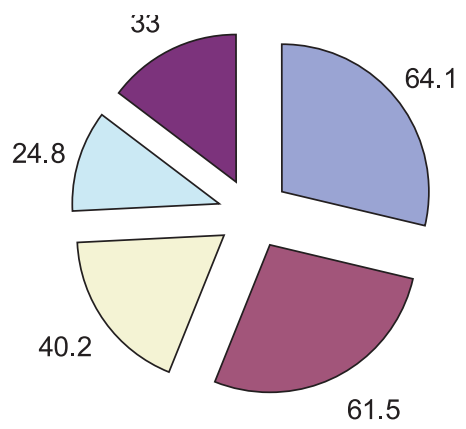

$\square$ HTN $\square$ Dyslipidemia $\square$ Diabetese Mellitus $\square$ FH $\square$ Smoker (all Male)

Fig.-2: Percentage of Distribution of CAD Risk Factors

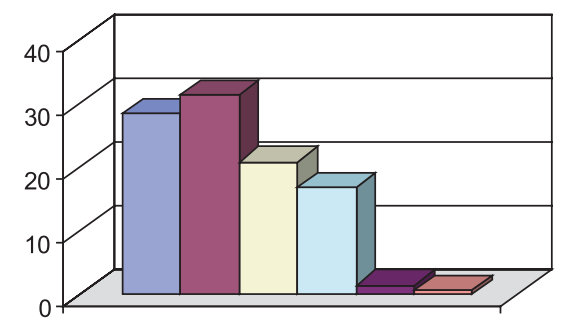

\begin{tabular}{|l|c|}
\hline$\square$ BMS & 28.5 \\
\hline$\square$ Sirolimus & 31.5 \\
\hline$\square$ Paclitaxel & 21 \\
\hline$\square$ Everolimus & 16.9 \\
\hline$\square$ Biolimus & 1.5 \\
\hline
\end{tabular}

Fig.-3.: Percentage of distribution of Types of Stent used

Fig 1. Shows the percentage distribution of the stented territory LAD 51 (43.6\%) followed by RCA 41 (35\%) and LCX 27(23\%). Average length and diameter of stented vessel were almost same in all territory. Territory wise multiple or overlapping stenting was done in LAD 3 (6 stents), RCA 1(2stents), LCX 4 (10 stents). Fig. 2. Showing the percentage distribution of $\mathrm{CAD}$ risk factors. Among the study group; 72 (61.5\%) were Dyslipidemic, 75 (64.1\%) were hypertensive: $47(40.2 \%)$ patients were Diabetic, FH 29 (24.8\%) and 33(33\%) were all male smoker. Fig 3. shows the percentage distribution of common stent used. Among the Stent used: BMS 37 (28.5\%), Sirolimus 41(31.5\%), Paclitaxel 27 (21\%), Everolimus 22 (16.9\%), Biolimus 2 (1.5\%) and Zotarolimus 1(0.75\%). Less Procedural time, reduced radiation exposure and no procedural complication were observed.

\section{Discussion:}

The purpose of the study was to evaluate the Transradial approach for PCI in our population subset at our center. Trans-radial PCI has gain popularity and being practiced in many of the centers worldwide. Be cause of the benefits include of patient comfort, early mobilization facilitate day case procedures and cost reduction. ${ }^{5-6}$

In most, the radial artery diameter is $2.5-3 \mathrm{~mm}$ and so it is compatible with $6 \mathrm{~F}$ or Larger. In a small proportion of patient specially female, short stature and Diabetes patient, the artery is of smaller caliber and may require a small sheath and catheter. ${ }^{7}$ 
Tortuous and small in caliber radial artery are prone to develop spasm and are common cause of procedural failure even for experienced operators. Vasovagal reaction, RA dissection and branch perforation, forearm haematoma, fistulae are not uncommon complications of RA intervention.

Pre-procedural cocktail of Heparin, Diltiazem or verapamil, Nitroglycerine and downsizing the catheter can reduce the RA spasm. Uses of long sheaths can minimize spasm related to manipulation and torqing of catheter during RA PCI. ${ }^{8}$ The need for the small guide catheters has been felt to be a significant limitation to the transradial approach because of poor visualization and inadequate backup support. ${ }^{9}$

In patient with ACS, PCI via TFA was associated with increase incidence of access site complication are common. ${ }^{10-12}$ These complications are an important cause of increased patient morbidity, longer hospital stay and higher hospital cost. ${ }^{13}$ The superficial location of RA allows easy homeostasis with less bleeding complication. ${ }^{14}$

Recently, the growing concerns of increased radiation exposure in trans-radial approach PCI is also debatable ${ }^{15}$ and can be alleviate by operators expertise in minimizing the cine-shot. We have presented this non-randomized prospective cohort of PCI by using trans-radial approach. In our present study, we found that females are more obese and developed coronary artery disease in advanced age. Common stented territory was LAD followed by RCA and LCX. Longer size stent was deployed in LAD, followed by RCA and LCX. BMS stent were deployed in $28.5 \%$ patient. Among the DES, Sirolimus was deployed in $31.5 \%$, followed by Paclitaxel stents and other limus.

In our present study, procedural failure and complication is negligible. Dehghani et $\mathrm{al}^{16}$ demonstrated that patient $>75$ yrs, prior CABG and short stature are common candidate for procedural failure.

\section{Conclusion:}

We may conclude that the radial approach for coronary procedure appears as safe and alternate to femoral access ${ }^{17}$ not only in routine and elective procedure, but also, in Primary PCI as well as in endovascular intervention at our center. Our study has revealed that trans-radial PCI is safe with reduced radiation exposure, quick mobilization of patient and no procedural complication in all the cases. It can be done routinely as an alternative to conventional Trans-femoral approach. Needless to say, that radial access virtually eliminates local vascular complication. With the advent of technical support, available hard ware and overall individual interventionist skills and well timed decision or measures in tackling procedural complication can make more success which will be evident throughout the year.

\section{References:}

1. Freestone B, Nolan J et al. Transradial cardiac procedures: the state of the art. Heart 2010;96:883-891.

2. Campeau L. Percutaneous radial artery approach for coronary angiography. Cath Cardiovasc Diag 1989;16:3-7

3. Eccleshall SC, Banks M, Caroll R. Implementation of diagnostic and interventional transradial program: resource and organizational implications. Heart 2003; 89:561-562.

4. Jolly SS, Amlani S, Hamon M. Radial versus femoral access for coronary angiography and intervention and the impact on major bleeding and ischemic events. Am Heart J 2009;157:132-140.

5. Cooper J, El-Sheikh RA, Cohen DJ. Effect of transradial access on quality of life and cost of cardiac catheterization. Am Heart J 1999;138:430-436.

6. Mann T, Cubeddu G, Schneider J et al. Right radial access for PCI; A prospective study demonstrates reduced complications and hospital charges. J Inv Cardiol 1996(Suppl D):30-35.

7. Lo TS, Nolan J, Fountjopoulos E. Radial artery anomaly and its influence on transradial coronary procedural outcome. Heart 2009; 95:410-415

8. Mann T, Cubeddu G, Bowen J et al. Stenting in acute coronary syndromes: a comparison of radial versus femoral access sites. J Am Coll Cardiol 1998:32:572576.

9. Feldman T. Ulnar and radial coronary interventions: distal reaches of arterial access. Cath Cardiovasc Diag $1997 ; 41: 131$

10. Agiirre FV, Topol EJ Ferguson JJ. Bleeding complications with the chimeric antibody to platelet GP IIb/IIIa integrin in patient undergoing Percutaneous coronary intervention. Circulation 1995; 91:2890-2892. 
11. Pompa J, Saller L, Pichard A. Vascular complications after balloon and new device angioplasty. Circulation 1993; 88:1569-1578.

12. Kereiakes M, Klieiman A, Ambose A. Randomized double-blind placebo-controlled dose ranging study of tirofiban platelet IIb/IIIa blockade in high risk patient undergoing coronary angioplasty. J Am Coll Cardiol 1996; 27:536-562

13. Ellis SG, Miller DP, Brown KJ. In-hospital cost of Percutaneous coronary revascularization: critical determinants and implication. Circulation 1995;92:741-747.
14. Arnold A. Hemostasis after radial artery cardiac catheterization. J Inv Card 1996; 8(suppl) :26-29.

15. Mercui M, Mehta S, Xie C. Radial artery access as a predictor of increased radiation exposure during a diagnostic cardiac catheterization procedure $J \mathrm{Am}$ Coll Cardiol Intervention 2011;4:347-52

16. Dehghani P, Mohammad A, Bajaj R et al. Mechanism and predictors of failed transradial approach for percutaneous coronary interventions. $\mathrm{J} \mathrm{Am}$ Coll Cardiol Intervention 2009; 2:1057-1064.

17. Agostoni P, Biondi-Zoccai,GL, De Benedicts L et al. Radial versus femoral; approach for Percutaneous coronary diagnostics and interventional procedures. $J$ Am Coll Cardiol 2004; 44:349-356. 